\title{
Products of Factorial Schur Functions
}

\author{
Victor Kreiman \\ Department of Mathematics \\ University of Georgia \\ Athens, GA 30602 USA \\ vkreiman@math.uga.edu
}

Submitted: Mar 9, 2008; Accepted: Jun 10, 2008; Published: Jun 20, 2008

Mathematics Subject Classifications: 05E05, 05E10

\begin{abstract}
The product of any finite number of factorial Schur functions can be expanded as a $\mathbb{Z}[\mathbf{y}]$-linear combination of Schur functions. We give a rule for computing the coefficients in such an expansion. This rule generalizes the classical LittlewoodRichardson rule and several special cases of the Molev-Sagan rule.
\end{abstract}

\section{Introduction}

Let $\mathcal{P}_{n}$ denote the set of partitions $\left\{\lambda=\left(\lambda_{1}, \ldots, \lambda_{n}\right) \in \mathbb{N}^{n} \mid \lambda_{1} \geq \cdots \geq \lambda_{n}\right\}$, and for $\lambda \in \mathcal{P}_{n}$, let $\mathcal{T}_{\lambda}$ denote the set of all semistandard Young tableaux of shape $\lambda$ with entries in $\{1, \ldots, n\}$. Let $x=\left(x_{1}, \ldots, x_{n}\right)$ and $y=\left(y_{1}, y_{2}, \ldots\right)$ be two sets of variables. The Schur function $s_{\lambda}(x)$ and factorial Schur function $s_{\lambda}(x \mid y)$ are defined as follows:

$$
s_{\lambda}(x)=\sum_{T \in \mathcal{T}_{\lambda}} \prod_{a \in T} x_{a} \quad \text { and } \quad s_{\lambda}(x \mid y)=\sum_{T \in \mathcal{T}_{\lambda}} \prod_{a \in T}\left(x_{a}+y_{a+c(a)-r(a)}\right)
$$

where for entry $a \in T, c(a)$ and $r(a)$ are the column and row numbers of $a$ respectively. Note that if $y$ is specialized to $(0,0, \ldots)$, then $s_{\lambda}(x \mid y)=s_{\lambda}(x)$. Factorial Schur functions are special cases of double Schubert polynomials [LS1, LS2] (see [La1] for discussion). The factorial Schur function $s_{\lambda}(x \mid y)$, with $y$ specialized to $(0,-1,-2,-3, \ldots)$, was first defined by Biedenbarn and Louck [BL1, BL2], and further studied by Chen and Louck [CL]. The factorial Schur function $s_{\lambda}(x \mid y)$, as defined above, is due to Macdonald [Ma2] and Goulden and Greene [GG]. Factorial Schur functions appear in the study of the center of the enveloping algebra $U\left(\mathfrak{g l}_{n}\right)$ (see Okounkov [Ok], Okounkov and Olshanski [OO], Nazarov [Na], Molev [Mo2, Mo1], and Molev and Sagan [MS]), and the equivariant cohomology of the Grassmannian (see Knutson and Tao [KT], Molev [Mo1], and Kreiman $[\mathrm{Kr}])$. 
Let $r \in \mathbb{N}$. For $i \in\{1, \ldots, r\}$, let $y^{(i)}=\left(y_{1}^{(i)}, y_{2}^{(i)}, \ldots\right)$ be an infinite set of variables, and let $\mathbf{y}$ denote the set of variables $\left(y^{(1)}, \ldots, y^{(r)}\right)$. As $\lambda$ varies over $\mathcal{P}_{n}$, both the Schur functions $s_{\lambda}(x)$ and the factorial Schur functions $s_{\lambda}\left(x \mid y^{(i)}\right)$ (where $i \in\{1, \ldots, r\}$ is fixed) form $\mathbb{Z}[\mathbf{y}]$-bases for $\mathbb{Z}[x, \mathbf{y}]^{S_{n}}$, the ring of polynomials in the $x$ and $\mathbf{y}$ variables which are symmetric in the $x$ variables. Hence for any sequence $\boldsymbol{\lambda}=\left(\lambda^{(1)}, \ldots, \lambda^{(r)}\right)$ of elements of $\mathcal{P}_{n}$, the polynomial $s_{\boldsymbol{\lambda}}(x \mid \mathbf{y})=s_{\lambda^{(1)}}\left(x \mid y^{(1)}\right) \cdots s_{\lambda^{(r)}}\left(x \mid y^{(r)}\right)$ can be expanded as a $\mathbb{Z}[\mathbf{y}]$ linear combination of Schur functions:

$$
s_{\boldsymbol{\lambda}}(x \mid \mathbf{y})=\sum_{\mu \in \mathcal{P}_{n}} c_{\boldsymbol{\lambda}}^{\mu}(\mathbf{y}) s_{\mu}(x), \quad \text { for some } c_{\boldsymbol{\lambda}}^{\mu}(\mathbf{y}) \in \mathbb{Z}[\mathbf{y}] .
$$

We give a rule for computing the coefficients $c_{\boldsymbol{\lambda}}^{\mu}(\mathbf{y})$ for arbitrary $\boldsymbol{\lambda}$.

When $y^{(1)}=\cdots=y^{(r)}=(0,0, \ldots)$, the coefficients $c_{\boldsymbol{\lambda}}^{\mu}(\mathbf{y})$ are classical LittlewoodRichardson coefficients. Various rules for these coefficients are well known (see, for example, [Ma1, (4.38')], [RS, Corollary 6]). The following special cases of our rule coincide with special cases of Molev-Sagan [MS, Theorem 3.1]: $r=2$ and $y^{(1)}=(0,0, \ldots) ; r=2$ and $y^{(1)}=y^{(2)}=(0,0, \ldots)$; and $r=1$. The third of these special cases, namely $r=1$, gives the change of $\mathbb{Z}[\mathbf{y}]$-basis coefficients between the Schur and factorial Schur functions. Another tableau-based rule for these change of basis coefficients is given by Molev [Mo1], and a determinantal formula is given by Macdonald [Ma2]. More generally, change of basis coefficients between Schubert and double Schubert polynomials were obtained by Lascoux (see [La3, Theorem 10.2.6] and Macdonald [Ma1, (6.3) and (6.7)]).

A related problem is to expand $s_{\boldsymbol{\lambda}}(x \mid \mathbf{y})$ in the basis of factorial Schur functions $\left\{s_{\mu}\left(x \mid y^{(1)}\right), \mu \in \mathcal{P}_{n}\right\}$. A solution to this problem for $r=2$ was given by Molev-Sagan [MS, Theorem 3.1]. A solution for $r=2$ and $y^{(1)}=y^{(2)}$ which is positive in the sense of Graham [Gr] was given by Knutson and Tao [KT]. Knutson and Tao's rule is expressed in terms of puzzles. Molev [Mo1] and Kreiman [Kr] give a tableau-based rule which is equivalent to the Knutson-Tao puzzle rule.

The proof of our rule for $c_{\boldsymbol{\lambda}}^{\mu}(\mathbf{y})$ generalizes a concise proof by Stembridge [St] of the classical Littlewood-Richardson rule. Stembridge's proof relies on sign-reversing involutions on skew tableaux which were introduced by Bender and Knuth [BK]. We generalize these arguments and constructions to barred skew tableaux, which are refinements of skew tableaux. Our proof is similar to but simpler than the proof used in [Kr], where Stembridge's methods are generalized to hatted skew tableaux.

Acknowledgements. We thank W. Graham and A. Molev for helpful comments and discussions. We also thank the referee for useful suggestions and remarks.

\section{Computing the Coefficients $c_{\lambda}^{\mu}(\mathbf{y})$}

An element $\lambda=\left(\lambda_{1}, \ldots, \lambda_{n}\right) \in \mathcal{P}_{n}$ can also be regarded as the Young diagram whose $i$-th row has $\lambda_{i}$ boxes. As above, let $\boldsymbol{\lambda}=\left(\lambda^{(1)}, \ldots, \lambda^{(r)}\right)$ be a sequence of elements in $\mathcal{P}_{n}$, which we regard as a sequence of Young diagrams. Denote also by $\boldsymbol{\lambda}$ the skew diagram formed by placing each Young diagram in the sequence $\lambda^{(1)}, \ldots, \lambda^{(r)}$ below and to the left of the 
preceding one. A barred skew tableau $T$ of shape $\boldsymbol{\lambda}$ is a filling of the boxes of the skew diagram $\boldsymbol{\lambda}$ with elements of $\{1, \ldots, n\} \cup\{\overline{1}, \ldots, \bar{n}\}$ in such a way that the entries weakly increase along any row from left to right and strictly increase along any column from top to bottom, without regard to whether or not the entries are barred.

The unbarred column word of $T$ is the sequence of unbarred entries of $T$ obtained by beginning at the top of the rightmost column, reading down, then moving to the top of the next to rightmost column and reading down, etc. (the barred entries are just skipped over in this process). We say that the unbarred column word of $T$ is Yamanouchi if, when one writes down its entries and stops at any point, one will have written at least as many ones as twos, at least as many twos as threes, etc. The unbarred content of $T$ is $\omega(T)=\left(\xi_{1}, \ldots, \xi_{n}\right) \in \mathbb{N}^{n}$, where $\xi_{k}$ is the number of unbarred $k$ 's in $T$. Define $c_{T}(\mathbf{y})=\prod y_{a+c(a)-r(a)}^{(i(a))}$, where the product is over all barred $a \in T, i(a)$ is the index of the particular Young diagram in which $a$ resides, and $c(a)$ and $r(a)$ denote the column and row numbers of $a$ in this Young diagram.

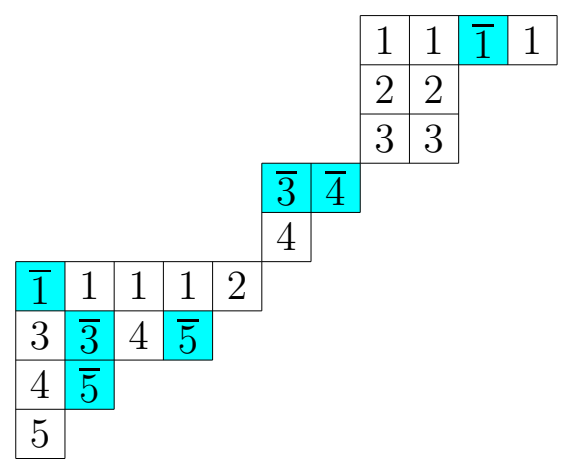

Figure 1: A barred skew tableau $T$ of shape $\boldsymbol{\lambda}$ and unbarred content $\mu$, where $\boldsymbol{\lambda}=$ $((4,2,2),(2,1),(5,4,2,1))$, and $\mu=\omega(T)=(6,3,3,3,1)$. The unbarred column word of $T$ is 1123123421141345 , which is Yamanouchi. Thus $T \in \mathcal{L} \mathcal{R}_{\boldsymbol{\lambda}}^{\mu}$. We have $c_{T}(\mathbf{y})=$ $\prod_{a \in T, a \text { barred }} y_{a+c(a)-r(a)}^{(i(a))}=y_{1+3-1}^{(1)} y_{3+1-1}^{(2)} y_{4+2-1}^{(2)} y_{1+1-1}^{(3)} y_{3+2-2}^{(3)} y_{5+4-2}^{(3)} y_{5+2-3}^{(3)}=y_{3}^{(1)} y_{3}^{(2)} y_{5}^{(2)}$ $y_{1}^{(3)} y_{3}^{(3)} y_{7}^{(3)} y_{4}^{(3)}$.

Definition 2.1. Denote the set of all barred skew tableaux of shape $\boldsymbol{\lambda}$ by $\mathcal{B}_{\boldsymbol{\lambda}}$, and the set of all barred skew tableaux of $\mathcal{B}_{\boldsymbol{\lambda}}$ of unbarred content $\mu$ whose unbarred column words are Yamanouchi by $\mathcal{L R}_{\lambda}^{\mu}$.

Theorem 2.2. $c_{\boldsymbol{\lambda}}^{\mu}(\mathbf{y})=\sum_{T \in \mathcal{L} \mathcal{R}_{\lambda}^{\mu}} c_{T}(\mathbf{y})=\sum_{T \in \mathcal{L} \mathcal{R}_{\lambda}^{\mu}}\left(\prod_{\substack{a \in T \\ a b a r r e d}} y_{a+c(a)-r(a)}^{(i(a))}\right)$.

Example 2.3. Let $n=2, \boldsymbol{\lambda}=((2,1),(1,1))$, and $\mu=(2,2)$. We list all $T \in \mathcal{L R}_{\boldsymbol{\lambda}}^{\mu}$, and for each $T$ we give $c_{T}(\mathbf{y})$ : 


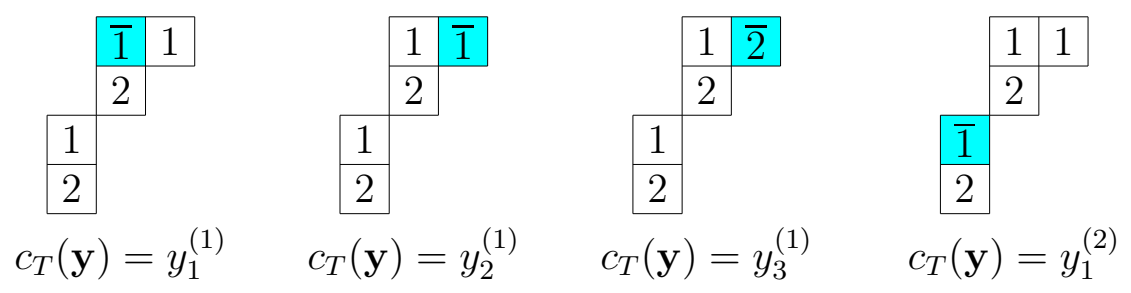

By Theorem 2.2, $c_{\boldsymbol{\lambda}}^{\mu}(\mathbf{y})=y_{1}^{(1)}+y_{2}^{(1)}+y_{3}^{(1)}+y_{1}^{(2)}$.

Remark 2.4. If $y^{(i)}$ is specialized to $(0,0, \ldots)$ for some $i$, then $s_{\lambda^{(i)}}\left(x \mid y^{(i)}\right)=s_{\lambda^{(i)}}(x)$. In this case, if $T \in \mathcal{L} \mathcal{R}_{\boldsymbol{\lambda}}^{\mu}$ has a barred entry in Young diagram $\lambda^{(i)}$, then $c_{T}(\mathbf{y})=0$. Thus in Theorem 2.2, $c_{\boldsymbol{\lambda}}^{\mu}(\mathbf{y})$ is computed by summing over only those $T \in \mathcal{L R}_{\boldsymbol{\lambda}}^{\mu}$ with no barred entries in Young diagram $\lambda^{(i)}$.

Remark 2.5. For simplicity, we assume here that $y:=y^{(1)}=\cdots=y^{(r)}$, and we denote $\mathbf{y}$ by just $y$. Theorem 2.2 has the following representation-theoretic interpretation. Let $G=G L_{n}(\mathbb{C})$, and let $H=\mathbb{C}^{*} \times \mathbb{C}^{*} \times \cdots$. For $\lambda \in \mathcal{P}_{n}$, let $V_{\lambda}$ denote the irreducible $G$-representation of highest weight $\lambda$. For $m \in \mathbb{Z}[y]=\mathbb{Z}\left[y_{1}, y_{2}, \ldots\right]$ a monomial with coefficient 1 , let $L_{m}$ denote the $H$ representation with character $m$. For $\lambda \in \mathcal{P}_{n}$, define the following $G \times H$ representation:

$$
U_{\lambda}=\bigoplus_{\substack{\mu \in \mathcal{P}_{n} \\ T \in \mathcal{L} \mathcal{R}_{(\lambda)}^{\mu}}} V_{\mu} \otimes L_{c_{T}(y)}
$$

By Theorem 2.2, $\operatorname{Char}_{G \times H} U_{\lambda}=\sum_{\mu \in \mathcal{P}_{n}, T \in \mathcal{L}_{(\lambda)}^{\mu}} s_{\mu}(x) c_{T}(y)=s_{\mu}(x \mid y)$.

Let $R(G \times H)$ denote the polynomial representation ring of $G \times H$ (i.e., the subring of the full representation ring of $G \times H$ generated by the polynomial representations) and $R(H)$ the polynomial representation ring of $H$. Then $R(G \times H) \cong \mathbb{Z}[x]^{S_{n}} \otimes \mathbb{Z}[y]=$ $\mathbb{Z}[x, y]^{S_{n}}$, and $R(H) \cong \mathbb{Z}[y]$. Since $\left\{s_{\lambda}(x \mid y) \mid \lambda \in \mathcal{P}_{n}\right\}$ forms a $\mathbb{Z}[y]$-basis for $\mathbb{Z}[x, y]^{S_{n}}$, the classes $\left\{\left[U_{\lambda}\right] \mid \lambda \in \mathcal{P}_{n}\right\} \subset R(G \times H)$ form an $R(H)$-basis for $R(G \times H)$. Theorem 2.2 implies the following decomposition as $G \times H$ representations:

$$
U_{\lambda^{(1)}} \otimes \cdots \otimes U_{\lambda^{(t)}}=\bigoplus_{\substack{\mu \in \mathcal{P}_{\mu} \\ T \in \mathcal{L} \mathcal{R}_{\lambda}^{\mu}}} V_{\mu} \otimes L_{c_{T}(y)}
$$

Remark 2.6. Factorial Schur functions can be defined in terms of reverse Young tableaux instead of semistandard Young tableaux (see [Kr]). Beginning with this definition, and with several minor adjustments to the proofs, one can obtain a rule for $c_{\boldsymbol{\lambda}}^{\mu}(\mathbf{y})$ which is almost the same as that of Theorem 2.2, except that it is expressed in terms of reverse barred skew tableaux instead of barred skew tableaux, with appropriate adjustments to the indexing of columns and rows. This rule is similar in form to the factorial Littlewood-Richardson rule of Molev [Mo1] and Kreiman [Kr], which is equivalent to the Knutson-Tao rule [KT]. 


\section{Generalization of Stembridge's Proof}

In this section we prove Theorem 2.2. The underlying structure and logic of our proof follows Stembridge [St]. Our approach is to generalize Stembridge's methods from skew tableaux to barred skew tableaux.

For $\xi=\left(\xi_{1}, \ldots, \xi_{n}\right) \in \mathbb{N}^{n}$, define $a_{\xi}(x)=\operatorname{det}\left[\left(x_{i}\right)^{\xi_{j}}\right]_{1 \leq i, j \leq n}$. Let $\rho=(n-1, n-$ $2, \ldots, 0) \in \mathcal{P}_{n}$.

Lemma 3.1. $a_{\rho}(x) s_{\boldsymbol{\lambda}}(x \mid \mathbf{y})=\sum_{T \in \mathcal{B}_{\boldsymbol{\lambda}}} c_{T}(\mathbf{y}) a_{\rho+\omega(T)}(x)$.

Lemma 3.2. $\sum c_{T}(\mathbf{y}) a_{\rho+\omega(T)}(x)=0$, where the sum is over all $T \in \mathcal{B}_{\boldsymbol{\lambda}}$ such that the unbarred column word of $T$ is not Yamanouchi.

The proofs of these two lemmas appear at the end of this section. The following three corollaries are easy consequences.

Corollary 3.3. $a_{\rho}(x) s_{\boldsymbol{\lambda}}(x \mid \mathbf{y})=\sum c_{T}(\mathbf{y}) a_{\rho+\omega(T)}(x)$, where the sum is over all $T \in \mathcal{B}_{\boldsymbol{\lambda}}$ such that the unbarred column word of $T$ is Yamanouchi.

If we set $r=1, \boldsymbol{\lambda}=(\lambda)$, and $\mathbf{y}=((0,0, \ldots))$ in Corollary 3.3 , then $s_{\boldsymbol{\lambda}}(x \mid \mathbf{y})=s_{\lambda}(x)$, and there is a unique $T \in \mathcal{B}_{\boldsymbol{\lambda}}$ with no barred entries (so that $c_{T}(\mathbf{y}) \neq 0$; see Remark 2.4) whose unbarred column word is Yamanouchi: namely, the tableau whose $i$-th row contains all $i$ 's. For this $T, c_{T}(\mathbf{y})=1$ and $\omega(T)=\lambda$. Thus we obtain the following well known bialternant formula for the Schur function.

Corollary 3.4. $s_{\lambda}(x)=a_{\rho+\lambda}(x) / a_{\rho}(x)$.

Dividing both sides of the relation of Corollary 3.3 by $a_{\rho}(x)$ and applying Corollary 3.4 yields

Corollary 3.5. $s_{\boldsymbol{\lambda}}(x \mid \mathbf{y})=\sum c_{T}(\mathbf{y}) s_{\omega(T)}(x)$, where the sum is over all $T \in \mathcal{B}_{\boldsymbol{\lambda}}$ such that the unbarred column word of $T$ is Yamanouchi.

Regrouping terms in the summation, $s_{\boldsymbol{\lambda}}(x \mid \mathbf{y})=\sum_{\mu \in \mathcal{P}_{n}} \sum_{T \in \mathcal{L} \mathcal{R}_{\boldsymbol{\lambda}}^{\mu}} c_{T}(\mathbf{y}) s_{\mu}(x)$. This proves Theorem 2.2.

\section{Involutions on Barred Skew Tableaux}

The proofs of Lemmas 3.1 and 3.2 rely on involutions $s_{1}, \ldots, s_{n-1}$ on $\mathcal{B}_{\boldsymbol{\lambda}}$, which generalize the involutions on semistandard Young tableaux introduced by Bender and Knuth [BK]. We now define these involutions and prove several of their properties.

Let $T \in \mathcal{B}_{\boldsymbol{\lambda}}$, and let $i \in\{1, \ldots, n-1\}$ be fixed. Let $a$ be an entry of $T$. Then either $a=j$ or $a=\bar{j}$, where $j \in\{1, \ldots, n\}$. We call $j$ the value of $a$. We say that an entry of $T$ of value $i$ or $i+1$ is free if there is no entry of value $i+1$ or $i$ respectively in the same column; semi-free if there is an entry of value $i+1$ or $i$ respectively in the same column, and exactly one of the two is barred; or locked if there is an entry of value $i+1$ or $i$ respectively in the same column, and either both entries are unbarred or both entries are 
barred. Note that any entry of value $i$ or $i+1$ must be exactly one of these three types. In any row, the free entries are consecutive. Semi-free entries come in pairs, one below the other, as do locked entries.

The barred skew tableau $s_{i} T$ is obtained by applying Alorithm 1 to each semi-free pair of entries in $T$, and applying Algorithm 2 to each maximal string of consecutive free entries $S$ lying on the same row.

\section{Algorithm 1}

For a semi-free pair consisting of two entries lying in the same column of $T$, the bar is removed from the barred entry and placed on top of the unbarred entry.

\section{Algorithm 2}

Let $l$ be the number of unbarred $i$ 's and $r$ the number of unbarred $i+1$ 's that $S$ contains.

- If $l=r$ : Do not change $S$.

- If $l<r$ : If $l=0$ then define $R=S$. Otherwise, letting $S_{1}$ be the $l$-th $i$ of $S$ from the left and $S_{2}$ the l-th $i+1$ of $S$ from the right, define $R$ to be the string of consecutive entries of $S$ beginning with the first $i+1$ or $\overline{i+1}$ of $S$ to the right of $S_{1}$ and ending with the first $i+1$ of $S$ to the left of $S_{2}$. Note that each entry of $R$ is either an $i+1$ or $\overline{i+1}$. Modify $R$ as follows:

1. change each $i+1$ to an $i$ and each $\overline{i+1}$ to an $\bar{i}$; and then

2. beginning with the rightmost $\bar{i}$ and then proceeding to the left, swap each $\bar{i}$ with the $i$ immediately to the right of it.

- If $l>r$ : If $r=0$ then define $R=S$. Otherwise, letting $S_{1}$ be the $r$-th $i$ of $S$ from the left and $S_{2}$ the $r$-th $i+1$ of $S$ from the right, define $R$ to be the string of consecutive entries of $S$ beginning with the first $i$ of $S$ to the right of $S_{1}$ and ending with the first $i$ or $\bar{i}$ of $S$ to the left of $S_{2}$. Note that each entry of $R$ is either an $i$ or an $\bar{i}$. Modify $R$ as follows:

1. change each $i$ to an $i+1$ and each $\bar{i}$ to an $\overline{i+1}$; and then

2. beginning with the leftmost $\overline{i+1}$ and then proceeding to the right, swap each $\overline{i+1}$ with the $i+1$ immediately to the left of it.

One checks that $s_{i}$ is an involution on $\mathcal{B}_{\boldsymbol{\lambda}}$. Let $S_{n}$ denote the permutation group on $n$ elements and $\sigma_{i}$ the simple transposition of $S_{n}$ which exchanges $i$ and $i+1$. The following Lemma follows from the construction of $s_{i}$.

Lemma 3.6. Let $T \in \mathcal{B}_{\boldsymbol{\lambda}}$. Then

(i) $c_{s_{i} T}(\mathbf{y})=c_{T}(\mathbf{y})$.

(ii) $\omega\left(s_{i} T\right)=\sigma_{i} \omega(T)$. 
Let $T \in \mathcal{B}_{\boldsymbol{\lambda}}$ and let $\sigma \in S_{n}$. Choose some decomposition of $\sigma$ into simple transpositions: $\sigma=\sigma_{i_{1}} \cdots \sigma_{i_{t}}$, and define $\sigma T=s_{i_{1}} \cdots s_{i_{t}} T$. By Lemma 3.6,

$$
c_{\sigma T}(\mathbf{y})=c_{T}(\mathbf{y}) \quad \text { and } \quad \omega(\sigma T)=\sigma \omega(T) .
$$

In particular, although $\sigma T$ depends on the decomposition of $\sigma$, both $c_{\sigma T}(\mathbf{y})$ and $\omega(\sigma T)$ are independent of the decomposition.

\section{Proof of Lemmas 3.1 and 3.2}

Proof of Lemma 3.1. Expanding $s_{\boldsymbol{\lambda}}(x \mid \mathbf{y})$ into monomials:

$$
\begin{aligned}
s_{\boldsymbol{\lambda}}(x \mid \mathbf{y}) & =\prod_{i=1}^{r} s_{\lambda^{(i)}}\left(x \mid y^{(i)}\right) \\
& =\prod_{i=1}^{r} \sum_{T \in \mathcal{T}_{\lambda^{(i)}}} \prod_{a \in T}\left(x_{a}+y_{a+c(a)-r(a)}^{(i)}\right) \\
& =\prod_{i=1}^{r} \sum_{T \in \mathcal{B}_{\left(\lambda^{(i)}\right)}} \prod_{\substack{a \in T \\
a \text { unbarred }}} x_{a} \prod_{\substack{a \in T \\
a \text { barred } \\
(i(a))}} y_{a+c(a)-r(a)}^{(i)}=\sum_{T \in \mathcal{B}_{\boldsymbol{\lambda}}} \prod_{\substack{a \in T \\
a \text { unbarred }}} x_{a} \prod_{\substack{a \in T \\
a \text { barred }}} y^{(i(T)} c_{T}(\mathbf{y}) .
\end{aligned}
$$

Therefore

$$
\begin{aligned}
a_{\rho}(x) s_{\boldsymbol{\lambda}}(x \mid \mathbf{y}) & =\sum_{\sigma \in S_{n}} \sum_{T \in \mathcal{B}_{\lambda}} \operatorname{sgn}(\sigma) x^{\sigma(\rho)} x^{\omega(T)} c_{T}(\mathbf{y}) \\
& =\sum_{\sigma \in S_{n}} \sum_{T \in \mathcal{B}_{\lambda}} \operatorname{sgn}(\sigma) x^{\sigma(\rho)} x^{\omega(\sigma T)} c_{\sigma T}(\mathbf{y}) \\
& =\sum_{\sigma \in S_{n}} \sum_{T \in \mathcal{B}_{\lambda}} c_{T}(\mathbf{y}) \operatorname{sgn}(\sigma) x^{\sigma(\rho+\omega(T))}=\sum_{T \in \mathcal{B}_{\boldsymbol{\lambda}}} c_{T}(\mathbf{y}) a_{\rho+\omega(T)}(x) .
\end{aligned}
$$

The second equality follows from the fact that $\sigma$ is an involution on $\mathcal{B}_{\boldsymbol{\lambda}}$; thus as $T$ varies over $\mathcal{B}_{\boldsymbol{\lambda}}$, so does $\sigma T$. The third equality follows from (2).

Proof of Lemma 3.2. For $T \in \mathcal{B}_{\boldsymbol{\lambda}}$ and $j$ a positive integer, define $T_{<j}$ to be the barred skew tableau consisting of the columns of $T$ lying to the left of column $j$ (and similarly for $\left.T_{\leq j}, T_{>j}, T_{\geq j}\right)$.

We will call the $T \in \mathcal{B}_{\boldsymbol{\lambda}}$ for which $\omega\left(T_{\geq j}\right) \notin \mathcal{P}_{n}$ for some $j$ Bad Guys (i.e., $T$ is a Bad Guy if and only if its unbarred column word is not Yamanouchi). Let $T$ be a Bad Guy, and let $j$ be maximal such that $\omega\left(T_{\geq j}\right) \notin \mathcal{P}_{n}$. Having selected $j$, let $i$ be minimal such that $\omega\left(T_{\geq j}\right)_{i}<\omega\left(T_{\geq j}\right)_{i+1}$. Since $\omega\left(T_{>j}\right)_{i} \geq \omega\left(T_{>j}\right)_{i+1}$ (by the maximality of $j$ ), we must 
have $\omega\left(T_{>j}\right)_{i}=\omega\left(T_{>j}\right)_{i+1}$, and column $j$ of $T$ must have an unbarred $i+1$ but not an unbarred $i$. Thus

$$
\left(\rho+\omega\left(T_{\geq j}\right)\right)_{i}=\left(\rho+\omega\left(T_{\geq j}\right)\right)_{i+1} .
$$

Define $T^{*}$ to be the barred skew tableau of shape $\boldsymbol{\lambda}$ obtained from $T$ by replacing $T_{<j}$ by $s_{i}\left(T_{<j}\right)$. It is clear that $T^{*} \in \mathcal{B}_{\boldsymbol{\lambda}}$, and that $T \mapsto T^{*}$ defines an involution on the Bad Guys in $\mathcal{B}_{\boldsymbol{\lambda}}$. Furthermore, by Lemma 3.6(i), $c_{T^{*}}(\mathbf{y})=c_{T}(\mathbf{y})$. By Lemma 3.6(ii), $\omega\left(\left(T^{*}\right)_{<j}\right)=$ $s_{i} \omega\left(T_{<j}\right)$. By $(3), \rho+\omega\left(\left(T^{*}\right)_{\geq j}\right)=\rho+\omega\left(T_{\geq j}\right)=s_{i}\left(\rho+\omega\left(T_{\geq j}\right)\right)$. Consequently, $\rho+\omega\left(T^{*}\right)=$ $s_{i}(\rho+\omega(T))$, implying that $a_{\rho+\omega\left(T^{*}\right)}(x)=-a_{\rho+\omega(T)}(x)$. Therefore $c_{T^{*}}(\mathbf{y}) a_{\rho+\omega\left(T^{*}\right)}(x)=$ $-c_{T}(\mathbf{y}) a_{\rho+\omega(T)}(x)$. Thus the contributions to $\sum c_{T}(\mathbf{y}) a_{\rho+\omega(T)}(x)$ of two Bad Guys paired under $T \mapsto T^{*}$ cancel, and the contribution of any Bad Guy paired with itself is 0 .

\section{Change of Basis Coefficients}

In this section, let $x_{(n)}=\left(x_{1}, \ldots, x_{n}\right)$ and $y=\left(y_{1}, y_{2}, \ldots\right)$ be two sets of variables. Since $\left\{s_{\mu}\left(x_{(n)}\right) \mid \mu \in \mathcal{P}_{n}\right\}$ and $\left\{s_{\mu}\left(x_{(n)} \mid y\right) \mid \mu \in \mathcal{P}_{n}\right\}$ both form $\mathbb{Z}[y]$-bases for $\mathbb{Z}[x, y]^{S_{n}}$, we have change of basis formulas

$$
\begin{aligned}
s_{\lambda}\left(x_{(n)} \mid y\right) & =\sum_{\mu \in \mathcal{P}_{n}} c_{\lambda, n}^{\mu}(y) s_{\mu}\left(x_{(n)}\right), \quad \text { for some } c_{\lambda, n}^{\mu}(y) \in \mathbb{Z}[y] . \\
s_{\lambda}\left(x_{(n)}\right) & =\sum_{\mu \in \mathcal{P}_{n}} d_{\lambda, n}^{\mu}(y) s_{\mu}\left(x_{(n)} \mid y\right), \quad \text { for some } d_{\lambda, n}^{\mu}(y) \in \mathbb{Z}[y] .
\end{aligned}
$$

Since (4) is a special case of (1), Theorem 2.2 gives a rule for the coefficients $c_{\lambda, n}^{\mu}(y)$. The same rule, as well as a rule for the coefficients $d_{\lambda, n}^{\mu}(y)$, can be obtained from Molev-Sagan [MS, Theorem 3.1] (see also Molev [Mo1, Section 4], which gives a different tableau-based rule for $\left.c_{\lambda, n}^{\mu}(y)\right)$. In this section we use a formula by Macdonald [Ma2] for $c_{\lambda, n}^{\mu}(y)$, which appears as Proposition 4.1(i) below, in order to derive formulas for $d_{\lambda, n}^{\mu}(y)$, which appear in Proposition 4.1(ii) and (iii). All proofs in this section are replicas or modifications of proofs appearing in Macdonald [Ma3, Chapter 1]. We point out that all formulas in Proposition 4.1 can be deduced as special cases of formulas involving double Schubert polynomials due to Lascoux (see Lascoux [La3, Theorem 10.2.6] and Macdonald [Ma1, (6.3) and (6.7)].

Let $r$ be a nonnegative integer and $p$ a positive integer. The $r$-th elementary and complete symmetric polynomials in variables $y_{1}, \ldots, y_{p}$, denoted by $e_{r}\left(y_{(p)}\right)$ and $h_{r}\left(y_{(p)}\right)$ respectively, are defined by the following generating functions:

$$
\begin{aligned}
& E\left(y_{(p)}, t\right)=\prod_{i=1}^{p}\left(1+y_{i} t\right)=\sum_{r \geq 0} e_{r}\left(y_{(p)}\right) t^{r} . \\
& H\left(y_{(p)}, t\right)=\prod_{i=1}^{p}\left(1-y_{i} t\right)^{-1}=\sum_{r \geq 0} h_{r}\left(y_{(p)}\right) t^{r} .
\end{aligned}
$$

It follows that $e_{0}\left(y_{(p)}\right)=h_{0}\left(y_{(p)}\right)=1$, and $e_{r}\left(y_{(p)}\right)=0$ for $r>p$. For $r<0, e_{r}\left(y_{(p)}\right)$ and $h_{r}\left(y_{(p)}\right)$ are defined to be 0 . For $\lambda=\left(\lambda_{1}, \ldots, \lambda_{n}\right) \in \mathcal{P}_{n}$, let $m$ be any integer greater 
than or equal to $\lambda_{1}^{\prime}$, the number of columns of $\lambda$. Define $\lambda^{c}=\left(\lambda_{1}^{c}, \ldots, \lambda_{m}^{c}\right) \in \mathcal{P}_{m}$, the complementary partition to $\lambda$, by $\lambda_{i}^{c}=n-\lambda_{m+1-i}^{\prime}, i=1, \ldots, m$.

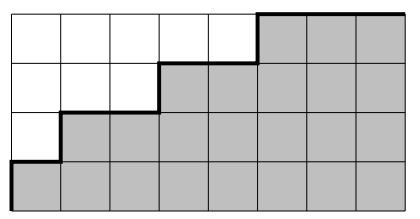

Figure 2: The partitions $\lambda=(5,3,1)$ and $\lambda^{c}=(4,4,4,3,3,2,2,1)$, where $n=4, m=8$.

Proposition 4.1. (i) $c_{\lambda, n}^{\mu}(y)=\operatorname{det}\left(e_{\lambda_{i}-\mu_{j}-i+j}\left(y_{\left(\lambda_{i}+n-i\right)}\right)\right)_{1 \leq i, j \leq n}$.

(ii) $d_{\lambda, n}^{\mu}(y)=\operatorname{det}\left(h_{\lambda_{i}-\mu_{j}-i+j}\left((-y)_{\left(\mu_{i}+n+1-i\right)}\right)\right)_{1 \leq i, j \leq n}$.

(iii) $d_{\lambda, n}^{\mu}(y)=c_{\mu^{c}, m}^{\lambda^{c}}(-y)$.

Before proving this proposition, we prove the following lemma (see also [Ma3, Chapter $1])$. Let $N \in \mathbb{N}, A=\left(e_{i-j}\left(y_{(i)}\right)\right)_{0 \leq i, j \leq N-1}$, and $B=\left(h_{i-j}\left((-y)_{(j+1)}\right)\right)_{0 \leq i, j \leq N-1}$.

Lemma 4.2. $A$ and $B$ are inverse matrices.

Proof. Let $q \geq p$. By $(6), E\left(y_{(q)}, t\right) H\left((-y)_{(p)}, t\right)$ is a polynomial of degree $q-p$ in $t$. Also by $(6)$,

$$
E\left(y_{(q)}, t\right) H\left((-y)_{(p)}, t\right)=\sum_{M \geq 0}\left(\sum_{r+s=M} e_{r}\left(y_{(q)}\right) h_{s}\left((-y)_{(p)}\right)\right) t^{M} .
$$

Thus for $q \geq p$,

$$
\sum_{r+s=M} e_{r}\left(y_{(q)}\right) h_{s}\left((-y)_{(p)}\right)=0, \text { if } M>q-p .
$$

For $0 \leq i, k \leq N-1$, consider $(A B)_{i, k}=\sum_{j=0}^{N-1} e_{i-j}\left(y_{(i)}\right) h_{j-k}\left((-y)_{(k+1)}\right)$. If $i<k$, then for each $j \in\{0, \ldots, N-1\}$, either $i-j<0$ or $j-k<0$; thus $(A B)_{i, k}=0$. If $i>k$, then $(A B)_{i, k}=0$ by $(7), M=i-k$. If $i=k$, then $(A B)_{i, k}=1$. This completes the proof.

Proof of Proposition 4.1. As noted above, (i) is proven in Macdonald [Ma2]. Define $\mathcal{P}_{n, m}=\left\{\nu \in \mathcal{P}_{n} \mid \nu_{1}^{\prime} \leq m\right\}$. Let $N=n+m$, and let $I_{n, N}$ denote the $n$-element subsets of $\{0, \ldots, N-1\}$, which we always assume are listed in increasing order. The map $\pi: \mathcal{P}_{n, m} \rightarrow I_{n, N}$ given by $\nu=\left(\nu_{i}\right)_{i=1}^{n} \mapsto I_{\nu}=\left\{\nu_{i}+n-i\right\}_{i=n}^{1}$, is a bijection.

The matrix $A$ is lower triangular with 1's along the diagonal, so $\operatorname{det}(A)=1$. For $I, J \in I_{n, N}$, let $A_{I, J}$ denote the $n \times n$ submatrix of $A$ with row set $I$ and column set $J$. By $(\mathrm{i}), c_{\lambda, n}^{\mu}(y)=\operatorname{det}\left(A_{I_{\lambda}, I_{\mu}}\right)$. This implies the following interpretation of $c_{\lambda, n}^{\mu}(y)$ : letting $\wedge^{n} A$ denote the $\left(\begin{array}{l}N \\ n\end{array}\right) \times\left(\begin{array}{l}N \\ n\end{array}\right)$ matrix $\left(\operatorname{det}\left(A_{I, J}\right)\right)_{I, J \in I_{n, N}}$, where the rows and columns of $\wedge^{n} A$ are ordered by some order on $I_{n, N}, c_{\lambda, n}^{\mu}=\left(\wedge^{n} A\right)_{I_{\lambda}, I_{\mu}}$ (note that $\left(\wedge^{n} A\right)_{I, J}$ refers to a single entry of $\wedge^{n} A$, whereas $A_{I, J}$ refers to an $n \times n$ submatrix of $A$ ). By Lemma 4.2, 
$\wedge^{n} B=\left(\wedge^{n} A\right)^{-1}$, and thus $d_{\lambda, n}^{\mu}(y)=\left(\wedge^{n} A\right)_{I_{\lambda}, I_{\mu}}^{-1}=\left(\wedge^{n} B\right)_{I_{\lambda}, I_{\mu}}=\operatorname{det}\left(B_{I_{\lambda}, I_{\mu}}\right)$. This proves (ii).

To prove (iii), we give a different expression for $\left(\wedge^{n} A\right)^{-1}$. For $I \in I_{n, N}$, define $\rho_{I}=$ $\#\left\{j<i \mid i \in I, j \in I^{\prime}\right\}$ and $I^{\prime}=\{0, \ldots, N-1\} \backslash I \in I_{m, N}$. The following formula gives the Laplace expansion for determinants (see, for example, [Bo, III, §8, no. 6]): for $I \in I_{n, N}, K \in I_{N-n, N}$

$$
\sum_{J \in I_{n, N}}(-1)^{\rho(I)+\rho(J)} \operatorname{det}\left(A_{I, J}\right) \operatorname{det}\left(A_{K, J^{\prime}}\right)=\left\{\begin{array}{ll}
\operatorname{det}(A) & \text { if } K=I^{\prime} \\
0 & \text { if } K \neq I^{\prime}
\end{array} .\right.
$$

Thus, since $\operatorname{det}(A)=1, \wedge^{n} A$ is invertible, and

$$
\left(\wedge^{n} A\right)^{-1}=\left((-1)^{\rho(I)+\rho(J)} \operatorname{det}\left(A_{J^{\prime}, I^{\prime}}\right)\right)_{I, J \in I_{n, N}} .
$$

Consequently,

$$
d_{\lambda, n}^{\mu}(y)=\left(\wedge^{n} A\right)_{I_{\lambda}, I_{\mu}}^{-1}=(-1)^{\rho\left(I_{\lambda}\right)+\rho\left(I_{\mu}\right)} \operatorname{det}\left(A_{I_{\mu}^{\prime}, I_{\lambda}^{\prime}}\right) .
$$

For $\nu \in \mathcal{P}_{n, m}$, consider the following two elementary properties of $I_{\nu}$.

$$
\text { 1. } \rho\left(I_{\nu}\right)=|\nu|-n \quad \text { 2. } I_{\nu}^{\prime}=I_{\nu^{c}}
$$

To prove property 1 , note that for $I_{\nu}=\left\{i_{1}, \ldots, i_{n}\right\}, i_{1}<\cdots<i_{n}$, we have $\rho\left(I_{\nu}\right)=$ $\left(i_{1}-1\right)+\cdots+\left(i_{n}-n\right)$ and $|\nu|=\left(i_{1}-0\right)+\cdots+\left(i_{n}-(n-1)\right)$. To prove property 2 , partition the rectangular Young diagram $D$ with $n$ rows and $m$ columns as $D=\nu \dot{\cup} \nu^{c}$ (see Figure 2). Number the boundary segments, which are darkened in Figure 2, from 0 to $m+n-1$. The numbers on the vertical and horizontal segments are the elements of $I_{\nu}$ and $I_{\nu^{c}}$ respectively. This completes the proof of the property 2 (see also [Ma3, (1.7)]).

Applying these two properties to (8),

$$
d_{\lambda, n}^{\mu}(y)=(-1)^{|\lambda|+|\mu|} \operatorname{det}\left(A_{I_{\mu^{c}}, I_{\lambda^{c}}}\right)=(-1)^{|\lambda|+|\mu|} c_{\mu^{c}, m}^{\lambda^{c}}(y) .
$$

Proposition 4.1(iii) now follows from the fact that $c_{\mu^{c}, m}^{\lambda^{c}}(y)$ is a homogeneous polynomial of degree $\left|\mu^{c}\right|-\left|\lambda^{c}\right|=(n m-|\mu|)-(n m-|\lambda|)=|\lambda|-|\mu|$; thus $(-1)^{|\lambda|+|\mu|} c_{\mu^{c}, m}^{\lambda^{c}}(y)=$ $(-1)^{|\lambda|-|\mu|} c_{\mu^{c}, m}^{\lambda^{c}}(y)=c_{\mu^{c}, m}^{\lambda^{c}}(-y)$.

Remark 4.3. Theorem 2.2 combined with Proposition 4.1 leads to a solution to the problem discussed in the introduction of expanding $s_{\boldsymbol{\lambda}}(x \mid \mathbf{y})$ in the basis of factorial Schur functions: $s_{\boldsymbol{\lambda}}(x \mid \mathbf{y})=\sum_{\mu \in \mathcal{P}_{n}} e_{\boldsymbol{\lambda}}^{\mu}(\mathbf{y}) s_{\mu}\left(x \mid y^{(i)}\right)$, where

$$
e_{\boldsymbol{\lambda}}^{\mu}(\mathbf{y})=\sum_{\nu \in \mathcal{P}_{n}} c_{\boldsymbol{\lambda}}^{\nu}(\mathbf{y}) d_{\nu, n}^{\mu}\left(y^{(i)}\right) .
$$

Additionally, one can express $c_{\boldsymbol{\lambda}}^{\nu}(\mathbf{y})$ in terms of change of basis coefficients and classical Littlewood-Richardson coefficients. For example, for $r=2$,

$$
c_{\boldsymbol{\lambda}}^{\mu}(\mathbf{y})=\sum_{\alpha, \beta \in \mathcal{P}_{n}} c_{\lambda^{(1)}, n}^{\alpha}\left(y^{(1)}\right) c_{\lambda^{(2)}, n}^{\beta}\left(y^{(2)}\right) c_{\alpha, \beta}^{\mu},
$$

where $c_{\alpha, \beta}^{\mu} \in \mathbb{Z}$ is the classical Littlewood-Richardson coefficient. 


\section{References}

[BK] E. A. Bender and D. E. Knuth, Enumeration of plane partitions, J. Combinatorial Theory Ser. A 13 (1972), 40-54.

[BL1] L. C. Biedenharn and J. D. Louck, A new class of symmetric polynomials defined in terms of tableaux, Adv. in Appl. Math. 10 (1989), no. 4, 396-438.

[BL2] - Inhomogeneous basis set of symmetric polynomials defined by tableaux, Proc. Nat. Acad. Sci. U.S.A. 87 (1990), no. 4, 1441-1445.

[Bo] N. Bourbaki, Algebra I. Chapters 1-3, Elements of Mathematics (Berlin), Springer-Verlag, Berlin, 1998, Translated from the French, Reprint of the 1989 English translation.

[CL] W. Y. C. Chen and J. D. Louck, The factorial Schur function, J. Math. Phys. 34 (1993), no. 9, 4144-4160.

[GG] I. Goulden and C. Greene, A new tableau representation for supersymmetric Schur functions, J. Algebra 170 (1994), no. 2, 687-703.

[Gr] W. Graham, Positivity in equivariant Schubert calculus, Duke Math. J. 109 (2001), no. 3, 599-614.

[Kr] V. Kreiman, Equivariant Littlewood-Richardson skew tableaux, arXiv:0706.3738.

[KT] A. Knutson and T. Tao, Puzzles and (equivariant) cohomology of Grassmannians, Duke Math. J. 119 (2003), no. 2, 221-260.

[La1] Alain Lascoux, Interpolation, Lectures at Tianjin University, June 1996.

[La2] A. Lascoux, Puissances extérieures, déterminants et cycles de Schubert, Bull. Soc. Math. France 102 (1974), 161-179.

[La3] - Symmetric functions and combinatorial operators on polynomials, CBMS Regional Conference Series in Mathematics, vol. 99, Published for the Conference Board of the Mathematical Sciences, Washington, DC, 2003.

[LR] D. E. Littlewood and A. R. Richardson, Group characters and algebra, Philos. Trans. Roy. Soc. London Ser. A 233 (1934).

[LS1] A. Lascoux and M.-P. Schützenberger, Polynômes de Schubert, C. R. Acad. Sci. Paris Sér. I Math. 294 (1982), no. 13, 447-450.

[LS2] - Structure de Hopf de l'anneau de cohomologie et de l'anneau de Grothendieck d'une variété de drapeaux, C. R. Acad. Sci. Paris Sér. I Math. 295 (1982), no. 11, 629-633.

[Ma1] I. G. Macdonald, Notes on schubert polynomials, Laboratoire de Combinatoire et d'Informatique Mathématique, vol. 6, Université du Québec à Montréal, 1991.

[Ma2] _ Schur functions: theme and variations, Séminaire Lotharingien de Combinatoire (Saint-Nabor, 1992), Publ. Inst. Rech. Math. Av., vol. 498, Univ. Louis Pasteur, Strasbourg, 1992, pp. 5-39. 
[Ma3] _ Symmetric functions and Hall polynomials, second ed., Oxford Mathematical Monographs, The Clarendon Press Oxford University Press, New York, 1995, With contributions by A. Zelevinsky, Oxford Science Publications.

[Mo1] A. I. Molev, Littlewood-Richardson polynomials, arXiv:0704.0065.

[Mo2] _ Factorial supersymmetric Schur functions and super Capelli identities, Kirillov's seminar on representation theory, Amer. Math. Soc. Transl. Ser. 2, vol. 181, Amer. Math. Soc., Providence, RI, 1998, pp. 109-137.

[MS] A. I. Molev and B. E. Sagan, A Littlewood-Richardson rule for factorial Schur functions, Trans. Amer. Math. Soc. 351 (1999), no. 11, 4429-4443.

[Na] M. Nazarov, Yangians and Capelli identities, Kirillov's seminar on representation theory, Amer. Math. Soc. Transl. Ser. 2, vol. 181, Amer. Math. Soc., Providence, RI, 1998, pp. 139-163.

[Ok] A. Okounkov, Quantum immanants and higher Capelli identities, Transform. Groups 1 (1996), no. 1-2, 99-126.

[OO] A. Okounkov and G. Olshanski, Shifted Schur functions, Algebra i Analiz 9 (1997), no. 2, 73-146, translation in St. Petersburg Math. J. 9 (1998), no. 2, 239-300.

[RS] J. B. Remmel and M. Shimozono, A simple proof of the Littlewood-Richardson rule and applications, Discrete Math. 193 (1998), no. 1-3, 257-266, Selected papers in honor of Adriano Garsia (Taormina, 1994).

[St] J. R. Stembridge, A concise proof of the Littlewood-Richardson rule, Electron. J. Combin. 9 (2002), no. 1, Note 5, 4 pp. (electronic). 